\title{
REMARKS ABOUT GEOMETRIC SCALE IN THE ANALYTIC HIERARCHY PROCESS
}

\author{
Tomasz Starczewski \\ Institute of Mathematics, Czestochowa University of Technology \\ Czestochowa, Poland \\ tomasz.starczewski@im.pcz.pl
}

Received: 22 August 2018; Accepted: 27 September 2018

\begin{abstract}
The Analytic Hierarchy Process (AHP) is perhaps the most popular approach to decision-making problems of prioritization. The basis of the AHP is pairwise comparison, which is used to compare alternatives. This comparisons are provided by decision makers usually as linguistic expressions which are next converted to numbers from a fixed set called a scale. The influence of the scale on the quality of prioritization was investigated in a number of papers. One of the most important types of judgment scale is the Geometric Scale. Its elements depend on specific parameters. In this paper, the impact of the choice of this scale's parameters on errors in priority vectors and on values of the inconsistency indices is studied via Monte Carlo simulations.
\end{abstract}

MSC 2010: 62C99, 90B50, 90C29, 90C31, $91 B 06$

Keywords: analytic hierarchy process, pairwise comparison, scale, inconsistency indices

\section{Analytic Hierarchy Process}

The Analytic Hierarchy Process (AHP) is a widely used method of multi-criteria decision analysis [1]. This method allows us to consider the multi-criteria decision problem in single-criteria parts. The decision maker (DM) compares each alternative with the others separately with respect to each criterion and each criterion with respect to the overriding goal. In this way a pairwise comparison matrix (PCM) arises. It is used to calculate the priority vector (PV), which collects relative weights of each alternative regarding criteria or the overriding goal. Finally, all priority vectors are joined to obtain priority weights of the alternatives reflecting the underlying preferences of DM [2].

In AHP practice, the DM judgments about priority ratio are expressed with the help of some linguistic phrases which are next converted to the numbers of a fixed set called a scale. Therefore the components of PCM are the numbers belong to a strictly specified set. 
The most popular scale consists of the first 9 natural numbers and their reciprocals: $\mathrm{FS}=\left\{\frac{1}{9}, \frac{1}{8}, \ldots, \frac{1}{2}, 1,2, \ldots, 9\right\}$. This original form is called the Fundamental Scale and was introduced by T. Saaty [3,4]. An extended version of this scale consists of more numbers (e.g 50 natural numbers and its reciprocal) and is called the Extended Scale. There are also other propositions of priority scales in literature [1,5-10]. One of them is the Geometric Scale (GS), whose elements form a geometric sequence. The commonly used version of GS consists of nine natural powers of 2 and their reciprocals, i.e.: $\mathrm{GS}=\left\{2^{-8}, 2^{-7} \ldots, 2^{-1}, 1,2, \ldots, 2^{8}\right\}$ or nine powers of $\sqrt{2}$ and reciprocals, i.e.: $\mathrm{GS}=\left\{2^{-4}, 2^{-3.5} \ldots, 2^{-0.5}, 1,2^{0.5}, \ldots, 2^{4}\right\}[1,6]$. However there are no restriction about power base or exponent range in GS. We test an impact of adopted GS's parameters on the correctness of the AHP procedure.

A scales usage inevitably leads to rounding errors. However, various scales may entail a different error's magnitude, so the important issue is the proper choice of the scale. Some relationships between scales and errors was the scope of other papers $[8,10,11]$, so in this article, we deal solely with various forms of the GS. For this paper we use computer simulation framework similar to the ones presented in literature [12-16].

An inconsistency analysis is an important part of AHP. It is argued that some PCMs cannot be used to estimate PVs because they contained judgment errors that were too large [3,17-19]. In order to eliminate such wrong matrices, the PCMquality characteristic was introduced. It is called an inconsistency index (ICI), but many authors proposed their own definitions [2, 16, 20-22]. In this paper, we also study the impact of the GS's parameters on the relationship between PV estimates errors and the ICIs.

\section{Preliminaries - notation}

The main procedure in AHP is PV's calculation. Assume that DM compares $n$ alternatives in respect to some criteria. Then we can mark a PV, which consists of priority weights corresponding to any criterion as: $\mathbf{w}=\left[w_{1}, w_{2}, \ldots, w_{n}\right]$. Therefore the PCM takes on the form:

$$
\mathbf{M}=\left[\begin{array}{ccccc}
1 & w_{1} / w_{2} & w_{1} / w_{3} & \ldots & w_{1} / w_{n} \\
w_{2} / w_{1} & 1 & w_{2} / w_{3} & \ldots & w_{2} / w_{n} \\
w_{3} / w_{1} & w_{3} / w_{2} & 1 & \ldots & w_{3} / w_{n} \\
\vdots & \vdots & \vdots & \ddots & \vdots \\
w_{n} / w_{1} & w_{n} / w_{2} & w_{n} / w_{3} & \ldots & 1
\end{array}\right]
$$

The judgments in the matrix $\mathbf{M}$ are called priority ratios. Because DM does not know "true" $\mathrm{PV}$, he/she usually is not able to estimate perfectly his/her priority ratios. Then 
we assume that the PCM's elements satisfy formula (e.g. [3, 16, 17, 19]):

$$
a_{i j}=\frac{w_{i}}{w_{j}} \cdot \varepsilon_{i j}
$$

in which $\varepsilon_{i j}$, called a perturbation factor, is the positive real random variable whose expected value $\mathrm{EX}=1$.

T. Saaty proposed to evaluate PV from PCM as a right eigenvector which is connected to the principal (i.e. maximal) eigenvalue of PCM [2]. This method is criticized for some drawbacks and disadvantages $[13,15,17,20]$. One of them is that this method can be used only for reciprocal matrices, i.e. for matrices whose elements located below diagonal are reciprocities of these located above diagonal:

$$
\forall i<j \quad a_{j i}=1 / a_{i j}
$$

Although the equation (3) is satisfied by priority ratios of formula (1), it usually does not happen for a DM matrices. In real life, reciprocity of PCM is enforced by replacement the components below diagonal by reciprocals of this above diagonal. Unfortunately, such a procedure results increase of errors in PCMs [15].

Popular procedure, which can be used to calculate PV's estimation for both reciprocal and non-reciprocal PCM, is so-called the Geometric Mean Method (GM) [20]. According to it, priority weight's estimation is expressed by the formula:

$$
v_{i}=\frac{\left(\prod_{j=1}^{n} a_{i j}\right)^{1 / n}}{\sum_{i=1}^{n}\left(\prod_{j=1}^{n} a_{i j}\right)^{1 / n}}, \quad i=1, \ldots, n 0
$$

The GM is promoted by some authors $[13,17,23,24]$. It is also easy to implement so we use it in our simulation.

Our paper is focused on GS parameters and theirs importance for AHP procedure. This scale, introduced by F. Lootsma [6], consists of powers of any real number. It is possible to use any real number as a power base, but number 2 or $\sqrt{2}$ are most often implemented. In opposite to FS, equal ratios characterize numbers of this scale. In our investigations we consider GS with variety power bases and a range of elements. We mark different cases of GS by the parameters in bracket (power base, elements number). Expression of scale elements, the numerical values of common ratios and the minimal and maximal elements of each scale are presented in Table 1 in Appendix.

\section{Inconsistency indices}

The important part of AHP is inconsistency analysis [16, 25]. DM's PCMs may contain some small inaccuracies or big errors. Because the true PVs are not known, it is not known if the errors in PCMs exist and how big they are. The manner to verify a correctness of PCMs is checking PCMs consistency. 
Definition 1 (The Consistent Pairwise Comparison Matrix). The pairwise comparison matrix $\mathbf{M}=\left[a_{i j}\right]_{n \times n}$ is called consistent if its elements satisfy condition:

$$
\forall i, j, k \quad a_{i k} \cdot a_{k j}=a_{i j}
$$

Indeed "ideal" PCMs, i.e. matrices which satisfy formula (1) simultaneously satisfy Definition 1. Regarding this fact, T. Saaty and the other authors introduce ICIs which are a "measure" of PCM's deviation from the "ideal" case. Although the values of ICIs indicate how much PCM is inconsistent, in reality they are threated as an indicator of the PCM-quality as a basis for PV's estimates. It would be a correct approach if they were strongly correlated with errors in PCMs and, maybe more importantly, with errors in estimated PVs [16,22]. This correlation depends on used scales, so we test the influence of GS's parameters on the quality of 4 presented below ICIs.

Definition 2 (Saaty's ICI). [2]. Let M is reciprocal PCM. Saaty's ICI of matrix M is given by the formula:

$$
\mathrm{SI}(n)=\frac{\lambda_{\max }-n}{n-1}
$$

where $\lambda_{\max }$ is the principal eigenvalue (i.e. maximal positive eigenvalue) of $\mathbf{M}$.

Definition 3 (Geometric ICI (by Crawford \& Williams)). [20]. Let $\mathbf{M}=\left[a_{i j}\right]_{n \times n}$ is a PCM and let $v_{i}(i=1, \ldots, n)$ are estimations of PV's components obtained by (4). Geometric ICI of $\mathbf{M}$ is given by the formula:

$$
\mathrm{GI}(n)=\frac{2}{(n-1)(n-2)} \sum_{i<j} \log ^{2}\left(\frac{a_{i j} v_{j}}{v_{i}}\right)
$$

Definition 4 (Koczkodaj's ICI). [21]. Let $\mathbf{M}=\left[a_{i j}\right]_{n \times n}$ is a PCM and let for any triad $\left(a_{i j}, a_{i k}, a_{k j}\right)$ of elements $\mathbf{M}$ a triad inconsistency is defined as:

$$
\operatorname{TI}\left(a_{i j}, a_{i k}, a_{k j}\right)=\min \left\{\left|1-\frac{a_{i j}}{a_{i k} a_{k j}}\right|,\left|1-\frac{a_{i k} a_{k j}}{a_{i j}}\right|\right\}
$$

Then the maximum of triads inconsistency is called Koczkodaj's ICI:

$$
\mathrm{KI}=\max \left(\mathrm{TI}\left(a_{i j}, a_{i k}, a_{k j}\right)\right)
$$

where the maximum is calculated for all different triads of matrix $\mathbf{M}$.

Definition 5 (Average Tridas ICI (by Grzybowski)). [16]. Let $\mathbf{M}=\left[a_{i j}\right]_{n \times n}$ is a PCM and let for any triad $\left(a_{i j}, a_{i k}, a_{k j}\right)$ of elements $\mathbf{M}$ a triad inconsistency is defined by formula (8). Then the average triads inconsistency we called Average Triad ICI:

$$
\mathrm{ATI}=\operatorname{Mean}\left(\mathrm{TI}\left(a_{i j}, a_{i k}, a_{k j}\right)\right)
$$


where the Mean is calculated for all different triads of matrix $\mathbf{M}$.

\section{Simulation framework}

In order to achieve the goal of the paper, we analyze errors in PCMs and PV's estimations and their correlation with ICIs. We take into account two kinds of errors: absolute (AE) and relative (RE) [16,22]:

$$
\begin{aligned}
& \operatorname{AE}(\mathbf{v}, \mathbf{w})=\sum_{i=1}^{n}\left|w_{i}-v_{i}\right| \\
& \operatorname{RE}(\mathbf{v}, \mathbf{w})=\sum_{i=1}^{n}\left|\frac{w_{i}-v_{i}}{w_{i}}\right|
\end{aligned}
$$

where $w_{i}(i=1, \ldots, n)$ are elements of a DM "true" PV and $v_{i}(i=1, \ldots, n)$ are their estimate. Analogously AE and RE for PCMs are defined.

Because we do not know in practice the DM's true PCM, we generate the "true" PV and connected with it the "perfect" PCM, which is next disturbed and rounded to scale. The estimation of PV is obtained on the basis of disturbed PCM. Our Monte Carlo simulations are similar to those existing in literature $[12,13,15,16,22,25]$ and run in following steps:

1. Generate random "true" $n$-dimensional PV and calculate "perfect" PCM (1).

2. Multiply a randomly chosen component above diagonal in "perfect" PCM by "big" factor $B \in D_{B}$.

3. Multiply all components of PCM by perturbation factor $\varepsilon_{i j}$ of a given probability distribution $\pi_{s}(2)$.

4. If the reciprocal matrix is under consideration, convert PCM components below diagonal by reciprocals of components above diagonal (3).

5. Round PCM components to selected scale (Table 1).

6. Calculate values of selected inconsistency indices on the basis of disturbed $\operatorname{PCM}(6,7,9,10)$.

7. Calculate the estimation of PV on the base of disturbed PCM. (4).

8. Calculate the AE and RE of PV estimation and of disturbed PCM in comparison to "true" PV and "perfect" PCM $(11,12)$.

9. Record all obtained values separately in the database. 
The "big" factor is the number from the set $D_{B}=\{1,2,3\}$ [16], wherein the number 1 means the lack of a "big" error. Distribution of the perturbation factor $\pi_{s}$ is one of the four: gamma, log-normal, truncated normal and uniform $[12,16,17,19,23]$, each used in the same number of matrices. We take into account 3 different standard deviations $\sigma_{\varepsilon} \in\{0.1,0.2,0.3\}[16,19]$. We consider both reciprocal and nonreciprocal PCMs to test the impact of "enforced" reciprocity in AHP.

The results which was presented in Table 2 show the values of AE and RE in disturbed PCMs and in comparison to them estimations of PVs. In the first four columns, the average errors are calculated on the basis of the PCMs disturbed evenly by a perturbation factor with standard deviation $\sigma_{\varepsilon}=0.2$. The 800 matrices were investigated (200 matrices for each distribution), each disturbed 25-times, so the average values were gained on the basis of 20000 PCMs and PV estimations. The next four columns present results for standard deviation of perturbation factor $\sigma_{\varepsilon} \in\{0.1,0.2,0.3\}$. Additionally multiplying by the "big" error $B=2$ was applied. The numbers in the Table are average values computed on the basis of 60000 PCMs or PV's estimations (20000 for each $\sigma_{\varepsilon}$ ). The investigation is made for both reciprocal and non-reciprocal PCMs.

The next characteristic which we use to test GS is the correlation coefficient between ICIs and errors in estimations of PV. In order to compute it, the disturbed PCMs are sorted regarding the values of ICIs and are split into some equal number classes. Then the average values of ICIs, AEs and REs related to each class are calculated and the Spearman Rank Correlation Coefficient (SRCC) on the basis of average values is computed [16]. The Tables 3-6 contain results for two different standard deviations of perturbation factor $\left(\sigma_{\varepsilon}=0.2\right.$ or $\left.\sigma_{\varepsilon}=0.3\right)$ as well as the results for joined case $\left(\sigma_{\varepsilon} \in\{0.1,0.2,0.3\}\right)$. The first three columns contain results for PCMs solely disturbed by the perturbation factor and the next three for PCMs with additional "big" error $B=3$. Similarly to Table 2 , the results are obtained on the basis of 20000 matrices (800 PCMs, each perturbed 25-times) and in joined case on the basis of 60000 matrices (2400 PCMs, each perturbed 25-times). The investigation was conducted for reciprocal and non-reciprocal matrices separately.

\section{Results}

The results collected in all tables in the Appendix are sorted similarly to scales in Table 1, i.e. by descending the range of scale elements and next by descending their ratio. One can see that errors in PCM and in PV estimations and the values of SRCC between errors and ICIs depend on GS's parameters.

When we look at Table 2, we observe the smallest values of the errors for scale $G S(\sqrt{2}, 16)$ or $G S(\sqrt{1.5}, 16)$ and the biggest errors for $G S(2,8)$ or $G S(\sqrt{2}, 8)$. One can note the scales with the same range of elements but with bigger elements number (smaller base) characterize the smaller errors in compare to this with a small number of elements. The scale range seems to have a big impact on the results. The smallest 
errors are usually observed for quite a big range, i.e. for $G S(\sqrt{1.5}, 16)$, whose the biggest element is $1.5^{8} \approx 25.63$.

The impact of GS's parameters on ICIs show Tables 3-6. Similar to Table 2, results are better for scales with a large number of elements (i.e. with small power base) but now the strongest correlation is observed for a small range of scale, e.g. for $G S(\sqrt[4]{2}, 16)$ or $G S(1.2,16)$. The SRCCs for the scales with big range (e.g. for $G S(2,8)$ or $G S(\sqrt{2}, 16))$ are the smallest among investigated scales. Especially for the reciprocal matrices and standard deviation $\sigma_{\varepsilon}=0.2$ it is usually negative.

When we compare the results in Tables 3-6, we can sometimes see a different magnitude of SRCC for different ICIs. The biggest disparity is for the index SI, for which the SRCC is high for reciprocal matrices but incomparably low for nonreciprocal ones. This cannot be observed for the other indices, for which the nonreciprocal PCMs with scale $G S(2,8)$ and $G S(\sqrt{2}, 16)$ achieve quite high correlation. For reciprocal matrices, we can note the lowest but acceptable value of SRCC for index KI. The highest SRCC is mostly demonstrated by index GI.

\section{Conclusions}

This paper shows the importance of GS's parameters proper choice in the AHP procedure. As one can see, the parameters have big impact on errors in PV's estimation as well as on the correlation between these errors and inconsistency indices. It turns out the commonly used GS, i.e. version $G S(2,8)$ or $G S(\sqrt{2}, 8)$, generate the highest errors in PCM and PV's estimation (among parameters investigated in our simulation). However doubling the number of elements in the scale results in reducing errors. The relationship is similar for errors in PCM and PV's estimation, however differ a little for correlation with indices. Weak correlation characterizes the scale $G S(2,8)$ and $G S(\sqrt{2}, 16)$ while the strong correlation characterizes the GS with a small maximal number e.g. $G S(1.2,16)$ or $G S(\sqrt[4]{2}, 16)$. In turn, the number of elements increasing cause usually better correlation with inconsistency indices and smaller values of errors in priorities estimation.

The important issue is the reciprocity of PCM. As one can see in Table 2, this artificial feature causes the big errors in PCMs and PV's estimations and worse correlation coefficients. Taking this into account, the better inconsistency indices seem to be GI or ATI among the investigated one.

In the light of our investigation, it seems reasonable to use in the AHP procedure the GS with a moderate range and high number of elements. While deciding about scale parameters it should be also take into account what is DM preference, whether they are more interested in small errors in preference weighs or strong correlation with inconsistency indices. 


\section{References}

[1] Ishizaka, A., \& Labib, A. (2011). Review of the main developments in the analytic hierarchy process. Expert Systems with Applications, 38(11), 14336-14345.

[2] Saaty, T.L. (1977). Scaling method for priorities in hierarchical structures. Journal of Mathematical Psychology, 15(3), 234-281.

[3] Saaty, T.L. (1980). The Analytic Hierarchy Process. NewYork: McGrawHill.

[4] Saaty, T.L. (2008). Decision making with the analytic hierarchy process. International Journal Services Sciences, 1(1), 83-98.

[5] Harker, P., \& Vargas, L. (1987). The Theory of Ratio Scale Estimation: Saaty's Analytic Hierarchy Process. Management Science, 33(11), 1383-1403.

[6] Lootsma, F. (1989). Conflict resolution via pairwise comparison of concessions. European Journal of Operational Research, 40, 109-116.

[7] Salo, A., \& Hamalainen, R. (1997). On the measurement of preference in the analytic hierarchy process. Journal of Multi-Criteria Decision Analysis, 6(6), 309-319.

[8] Dong, Y., Xu, Y., Li, H., \& Dai, M. (2008). A comparative study of the numerical scales and the prioritization methods in AHP. European Journal of Operational Research, 186, 229-242.

[9] Dong, Y., Hong, W.-C., Xu, Y., \& Yu, S. (2013). Numerical scales generated individually for analytic hierarchy process. European Journal of Operational Research, 229, 654-662.

[10] Franek, J., \& Kresta, A. (2014). Judgment scales and consistency measure in AHP. Procedia Economics and Finance, 12, 164-173.

[11] Starczewski, T. (2017). Remarks on the impact of the adopted scale on the priority estimation quality. Journal of Applied Mathematics and Computational Mechanics, 16(3), 105-116.

[12] Zahedi, F. (1986). A simulation study of estimation methods in the analytic hierarchy process. Socio-Economic Planning Sciences, 20, 347-354.

[13] Choo, E.U., \& Wedley, W.C. (2004). A common framework for deriving preference values from pairwise comparison matrices. Computers and Operations Research, 31, 893-908.

[14] Lin, C.-C. (2007). A revised framework for deriving preference values from pairwise comparison matrices. European Journal of Operational Research, 176, 1145-1150.

[15] Grzybowski, A.Z. (2012). Note on a new optimization based approach for estimating priority weights and related consistency index. Expert Systems with Applications, 39, 11699-11708.

[16] Grzybowski, A.Z. (2016). New results on inconsistency indices and their relationship with the quality of priority vector estimation. Expert Systems with Applications, 43, 197-212.

[17] Dijkstra, T.K. (2013). On the extraction of weights from pairwise comparison matrices. Central European Journal of Operations Research, 21, 103-123.

[18] Lin, C., Kou, G., \& Ergu, D. (2013). An improved statistical approach for consistency test in AHP. Annals of Operations Research, 211(1), 289-299.

[19] Starczewski, T. (2016). Relationship between priority ratios disturbances and priority estimation errors. Journal of Applied Mathematics and Computational Mechanics, 15(3), 143-154.

[20] Crawford, G., \& Williams, C.A. (1985). A note on the analysis of subjective judgment matrices. Journal of Mathematical Psychology, 29, 387-405.

[21] Koczkodaj, W.W. (1993). A new definition of consistency of pairwise comparisons. Mathematical and Computer Modelling, 18(7), 79-84.

[22] Kazibudzki, P.T. (2016). Redefinition of triad's inconsistency and its impact on the consistency measurement of pairwise comparison matrix. Journal of Applied Mathematics and Computational Mechanics, 15(1), 71-78. 
[23] Budescu, D.V., Zwick, R., \& Rapoport, A. (1986). Comparison of the analytic hierarchy process and the geometric mean procedure for ratio scaling. Applied Psychological Measurement, 10, 69-78.

[24] Aguaron, J., \& Moreno-Jimenez, J.M. (2003). The geometric consistency index: Approximated thresholds. European Journal of Operational Research, 147, 137-145.

[25] Kazibudzki, P.T. (2016). An examination of performance relations among selected consistency measures for simulated pairwise judgments. Annals of Operations Research, 244(2), 525-544. 


\section{Appendix}

Table 1. Geometric Scale with different parameters

\begin{tabular}{|l|c|c|c|c|}
\hline Scale & Components & Ratio & Min & Max \\
\hline $\mathrm{GS}(2,8)$ & $\left\{2^{-8}, 2^{-7} \ldots, 2^{-1}, 1,2, \ldots, 2^{8}\right\}$ & 2.00 & 0.004 & 256.0 \\
\hline $\mathrm{GS}(\sqrt{2}, 16)$ & $\left\{2^{-8}, 2^{-7.5} \ldots, 2^{-0.5}, 1,2^{0.5}, \ldots, 2^{8}\right\}$ & 1.41 & 0.004 & 256.0 \\
\hline $\mathrm{GS}(1.5,8)$ & $\left\{1.5^{-8}, 1.5^{-7} \ldots, 1.5^{-1}, 1,1.5, \ldots, 1.5^{8}\right\}$ & 1.50 & 0.039 & 25.6 \\
\hline $\mathrm{GS}(\sqrt{1.5}, 16)$ & $\left\{1.5^{-8}, 1.5^{-7.5} \ldots, 1.5^{-0.5}, 1,1.5^{0.5}, \ldots, 1.5^{8}\right\}$ & 1.23 & 0.039 & 25.6 \\
\hline $\mathrm{GS}(1.2,16)$ & $\left\{1.2^{-16}, 1.2^{-15} \ldots, 1.2^{-1}, 1,1.2, \ldots, 1.2^{16}\right\}$ & 1.20 & 0.054 & 18.5 \\
\hline $\mathrm{GS}(\sqrt{2}, 8)$ & $\left\{2^{-4}, 2^{-3.5} \ldots, 2^{-0.5}, 1,2^{0.5}, \ldots, 2^{4}\right\}$ & 1.41 & 0.063 & 16.0 \\
\hline $\mathrm{GS}(\sqrt[4]{2}, 16)$ & $\left\{2^{-4}, 2^{-3.75} \ldots, 2^{-0.25}, 1,2^{0.25}, \ldots, 2^{4}\right\}$ & 1.19 & 0.063 & 16.0 \\
\hline
\end{tabular}

Table 2. Average errors in PCM $5 \times 5$ rounded to variety GS and $P V$ estimated by GM for reciprocal and non-reciprocal matrices. Standard deviations of disturbance factor $\sigma_{\varepsilon}=0.2$ or joined $\sigma_{\varepsilon} \in\{0.1,0.2,0.3\}$

\begin{tabular}{|c|l|c|c|c|c|c|c|c|c|}
\hline \multicolumn{2}{|l|}{} & \multicolumn{4}{c|}{$\sigma_{\varepsilon}=0.2$} & \multicolumn{3}{c|}{$\sigma_{\varepsilon} \in\{0.1,0.2,0.3\}$} \\
\hline \multicolumn{2}{|c|}{ matrix } & \multicolumn{2}{c|}{ in PCM } & \multicolumn{2}{c|}{ in PV } & \multicolumn{2}{c|}{ in PCM } & \multicolumn{2}{c|}{ in PV } \\
\hline rec. & scale & \multicolumn{2}{|c|}{ AE } & \multicolumn{2}{c|}{ RE } & \multicolumn{2}{c|}{ AE } & \multicolumn{2}{c|}{ RE } \\
\hline yes & $G S(2,1)$ & 0.642 & 0.244 & 0.016 & 0.104 & 0.787 & 0.297 & 0.019 & 0.123 \\
\hline yes & $G S(\sqrt{2}, 16)$ & 0.496 & 0.187 & 0.012 & 0.079 & 0.653 & 0.247 & 0.017 & 0.109 \\
\hline yes & $G S(1.5,8)$ & 0.539 & 0.196 & 0.013 & 0.086 & 0.675 & 0.254 & 0.017 & 0.113 \\
\hline yes & $G S(\sqrt{1.5}, 16)$ & 0.496 & 0.178 & 0.012 & 0.076 & 0.637 & 0.236 & 0.017 & 0.108 \\
\hline yes & $G S(1.2,16)$ & 0.567 & 0.183 & 0.012 & 0.084 & 0.683 & 0.241 & 0.017 & 0.115 \\
\hline yes & $G S(\sqrt{2}, 8)$ & 0.612 & 0.201 & 0.014 & 0.094 & 0.750 & 0.259 & 0.018 & 0.124 \\
\hline yes & $G S(\sqrt[4]{2}, 16)$ & 0.599 & 0.188 & 0.013 & 0.092 & 0.713 & 0.245 & 0.018 & 0.121 \\
\hline no & $G S(2,8)$ & 0.592 & 0.225 & 0.014 & 0.087 & 0.694 & 0.264 & 0.016 & 0.102 \\
\hline no & $G S(\sqrt{2}, 16)$ & 0.481 & 0.179 & 0.011 & 0.069 & 0.592 & 0.222 & 0.013 & 0.087 \\
\hline no & $G S(1.5,8)$ & 0.516 & 0.186 & 0.011 & 0.074 & 0.632 & 0.230 & 0.014 & 0.092 \\
\hline no & $G S(\sqrt{1.5}, 16)$ & 0.505 & 0.172 & 0.010 & 0.070 & 0.576 & 0.212 & 0.013 & 0.086 \\
\hline no & $G S(1.2,16)$ & 0.574 & 0.179 & 0.011 & 0.079 & 0.645 & 0.218 & 0.014 & 0.094 \\
\hline no & $G S(\sqrt{2}, 8)$ & 0.571 & 0.192 & 0.012 & 0.083 & 0.709 & 0.236 & 0.015 & 0.105 \\
\hline no & $G S(\sqrt[4]{2}, 8)$ & 0.562 & 0.181 & 0.012 & 0.081 & 0.677 & 0.223 & 0.015 & 0.102 \\
\hline
\end{tabular}


Table 3. Spearman rank correlation coefficient between SI of disturbed rounded to scale PCM size $5 \times 5$ and $\mathrm{RE}$ in $\mathrm{PV}$ estimation related to this matrix

\begin{tabular}{|c|c|c|c|c|c|c|c|}
\hline \multirow{2}{*}{\multicolumn{2}{|c|}{ matrix }} & \multicolumn{6}{|c|}{$\mathrm{RE}$ in $\mathrm{PV}$} \\
\hline & & \multicolumn{3}{|c|}{$D B=1$} & \multicolumn{3}{|c|}{$D B=3$} \\
\hline rec. & scale & $\sigma_{\varepsilon}=0.2$ & $\sigma_{\varepsilon}=0.3$ & joined & $\sigma_{\varepsilon}=0.2$ & $\sigma_{\varepsilon}=0.3$ & joined \\
\hline yes & $G S(2,8)$ & -0.326 & 0.865 & 0.874 & -0.224 & 0.328 & 0.621 \\
\hline yes & $G S(\sqrt{2}, 16)$ & -0.096 & 0.820 & 0.991 & -0.069 & 0.265 & 0.708 \\
\hline yes & $G S(1.5,8)$ & 0.998 & 1 & 0.998 & 0.997 & 1 & 0.962 \\
\hline yes & $G S(\sqrt{1.5}, 16)$ & 1 & 0.998 & 1 & 1 & 1 & 0.995 \\
\hline yes & $G S(1.2,16)$ & 1 & 1 & 1 & 1 & 1 & 0.997 \\
\hline yes & $G S(\sqrt{2}, 8)$ & 1 & 1 & 1 & 0.992 & 1 & 0.986 \\
\hline yes & $G S(\sqrt[4]{2}, 16)$ & 1 & 1 & 1 & 1 & 1 & 0.998 \\
\hline no & $G S(2,8)$ & 0.024 & -0.279 & 0.119 & 0.817 & 0.500 & 0.382 \\
\hline no & $G S(\sqrt{2}, 16)$ & -0.406 & -0.408 & 0.047 & 0.844 & 0.265 & 0.147 \\
\hline no & $G S(1.5,8)$ & 0.628 & 0.570 & 0.215 & 0.994 & 0.982 & 0.643 \\
\hline no & $G S(\sqrt{1.5}, 16)$ & 0.595 & 0.643 & 0.176 & 0.998 & 0.992 & 0.368 \\
\hline no & $G S(1.2,16)$ & 0.770 & 0.574 & 0.320 & 0.998 & 0.997 & 0.519 \\
\hline no & $G S(\sqrt{2}, 8)$ & 0.772 & 0.764 & 0.365 & 1 & 0.986 & 0.690 \\
\hline no & $G S(\sqrt[4]{2}, 16)$ & 0.789 & 0.682 & 0.393 & 1 & 0.994 & 0.526 \\
\hline
\end{tabular}

Table 4. Spearman rank correlation coefficient between GI of disturbed rounded to scale PCM size $5 \times 5$ and $\mathrm{RE}$ in PV estimation related to this matrix

\begin{tabular}{|c|l|c|c|c|c|c|c|}
\hline \multicolumn{2}{|c|}{ matrix } & \multicolumn{6}{|c|}{ RE in PV } \\
\hline \multicolumn{2}{|c|}{} & \multicolumn{3}{c|}{$D B=1$} & \multicolumn{3}{c|}{$D B=3$} \\
\hline \hline rec. & scale & $\sigma_{\varepsilon}=0.2$ & $\sigma_{\varepsilon}=0.3$ & joined & $\sigma_{\varepsilon}=0.2$ & $\sigma_{\varepsilon}=0.3$ & joined \\
\hline \hline yes & $G S(2,8)$ & -0.209 & 0.719 & 0.829 & -0.417 & 0.374 & 0.578 \\
\hline yes & $G S(\sqrt{2}, 16)$ & -0.080 & 0.820 & 0.974 & -0.057 & 0.218 & 0.767 \\
\hline yes & $G S(1.5,8)$ & 1 & 1 & 1 & 0.997 & 1 & 0.977 \\
\hline yes & $G S(\sqrt{1.5}, 16)$ & 1 & 1 & 1 & 1 & 1 & 0.994 \\
\hline yes & $G S(1.2,16)$ & 1 & 1 & 1 & 1 & 1 & 0.997 \\
\hline yes & $G S(\sqrt{2}, 8)$ & 1 & 1 & 1 & 0.997 & 1 & 0.980 \\
\hline yes & $G S(\sqrt[4]{2}, 16)$ & 1 & 1 & 1 & 1 & 1 & 0.998 \\
\hline no & $G S(2,8)$ & 0.925 & 0.988 & 0.962 & 0.982 & 0.980 & 0.991 \\
\hline no & $G S(\sqrt{2}, 16)$ & 0.967 & 0.982 & 1 & 0.934 & 0.956 & 0.971 \\
\hline no & $G S(1.5,8)$ & 0.998 & 0.998 & 1 & 1 & 1 & 0.994 \\
\hline no & $G S(\sqrt{1.5}, 16)$ & 1 & 1 & 1 & 1 & 1 & 1 \\
\hline no & $G S(1.2,16)$ & 1 & 1 & 1 & 1 & 1 & 1 \\
\hline no & $G S(\sqrt{2}, 8)$ & 1 & 1 & 1 & 1 & 1 & 1 \\
\hline no & $G S(\sqrt[4]{2}, 16)$ & 1 & 1 & 1 & 1 & 1 & 1 \\
\hline
\end{tabular}


Table 5. Spearman rank correlation coefficient between KI of disturbed rounded to scale PCM size $5 \times 5$ and $\mathrm{RE}$ in $\mathrm{PV}$ estimation related to this matrix

\begin{tabular}{|c|l|c|c|c|c|c|c|}
\hline \multicolumn{2}{|c|}{ matrix } & \multicolumn{5}{c|}{$D B=1$} & \multicolumn{3}{c|}{$D B=3$} \\
\hline \hline rec. & scale & $\sigma_{\varepsilon}=0.2$ & $\sigma_{\varepsilon}=0.3$ & joined & $\sigma_{\varepsilon}=0.2$ & $\sigma_{\varepsilon}=0.3$ & joined \\
\hline \hline yes & $G S(2,8)$ & -0.132 & 0.537 & 0.592 & -0.330 & 0.025 & 0.168 \\
\hline yes & $G S(\sqrt{2}, 16)$ & 0.311 & 0.586 & 0.869 & -0.143 & 0.037 & 0.455 \\
\hline yes & $G S(1.5,8)$ & 0.922 & 0.959 & 0.932 & 0.960 & 0.974 & 0.885 \\
\hline yes & $G S(\sqrt{1.5}, 16)$ & 0.967 & 0.980 & 0.974 & 0.975 & 0.991 & 0.926 \\
\hline yes & $G S(1.2,16)$ & 0.975 & 0.980 & 0.992 & 0.988 & 0.987 & 0.954 \\
\hline yes & $G S(\sqrt{2}, 8)$ & 0.951 & 0.958 & 0.973 & 0.969 & 0.979 & 0.952 \\
\hline yes & $G S(\sqrt[4]{2}, 16)$ & 0.977 & 0.996 & 0.993 & 0.980 & 0.992 & 0.959 \\
\hline no & $G S(2,8)$ & 0.622 & 0.652 & 0.681 & 0.869 & 0.899 & 0.600 \\
\hline no & $G S(\sqrt{2}, 16)$ & 0.582 & 0.685 & 0.891 & 0.921 & 0.884 & 0.630 \\
\hline no & $G S(1.5,8)$ & 0.922 & 0.936 & 0.918 & 0.960 & 0.976 & 0.805 \\
\hline no & $G S(\sqrt{1.5}, 16)$ & 0.908 & 0.958 & 0.952 & 0.979 & 0.988 & 0.906 \\
\hline no & $G S(1.2,16)$ & 0.955 & 0.964 & 0.991 & 0.987 & 0.987 & 0.910 \\
\hline no & $G S(\sqrt{2}, 8)$ & 0.930 & 0.959 & 0.945 & 0.969 & 0.975 & 0.915 \\
\hline no & $G S(\sqrt[4]{2}, 16)$ & 0.978 & 0.966 & 0.992 & 0.974 & 0.991 & 0.931 \\
\hline
\end{tabular}

Table 6. Spearman rank correlation coefficient between ATI of disturbed rounded to scale PCM size $5 \times 5$ and RE in PV estimation related to this matrix

\begin{tabular}{|c|c|c|c|c|c|c|c|}
\hline & & \multicolumn{6}{|c|}{ RE in PV } \\
\hline \multicolumn{2}{|r|}{ matrix } & \multicolumn{3}{|c|}{$D B=1$} & \multicolumn{3}{|c|}{$D B=3$} \\
\hline rec. & scale & $\sigma_{\varepsilon}=0.2$ & $\sigma_{\varepsilon}=0.3$ & joined & $\sigma_{\varepsilon}=0.2$ & $\sigma_{\varepsilon}=0.3$ & joined \\
\hline yes & $G S(2,8)$ & -0.254 & 0.851 & 0.825 & -0.556 & 0.451 & 0.760 \\
\hline yes & $G S(\sqrt{2}, 16)$ & -0.126 & 0.755 & 0.967 & -0.220 & 0.247 & 0.959 \\
\hline yes & $G S(1.5,8)$ & 0.973 & 0.995 & 0.997 & 0.991 & 0.998 & 0.994 \\
\hline yes & $G S(\sqrt{1.5}, 16)$ & 1 & 1 & 0.998 & 1 & 1 & 1 \\
\hline yes & $G S(1.2,16)$ & 1 & 1 & 1 & 1 & 1 & 1 \\
\hline yes & $G S(\sqrt{2}, 8)$ & 0.992 & 0.998 & 0.997 & 1 & 1 & 0.998 \\
\hline yes & $G S(\sqrt[4]{2}, 16)$ & 1 & 1 & 1 & 1 & 1 & 1 \\
\hline no & $G S(2,8)$ & 0.726 & 0.893 & 0.845 & 0.935 & 0.947 & 0.955 \\
\hline no & $G S(\sqrt{2}, 16)$ & 0.823 & 0.919 & 0.964 & 0.919 & 0.967 & 0.995 \\
\hline no & $G S(1.5,8)$ & 0.970 & 0.992 & 0.995 & 0.971 & 1 & 0.989 \\
\hline no & $G S(\sqrt{1.5}, 16)$ & 0.997 & 0.998 & 0.998 & 1 & 1 & 1 \\
\hline no & $G S(1.2,16)$ & 1 & 1 & 1 & 1 & 1 & 1 \\
\hline no & $G S(\sqrt{2}, 8)$ & 0.982 & 0.998 & 0.997 & 0.998 & 0.992 & 0.995 \\
\hline no & $G S(\sqrt[4]{2}, 16)$ & 1 & 0.998 & 1 & 1 & 1 & 1 \\
\hline
\end{tabular}

